\title{
Feasibility of a User-Independent Systematic Method for Medical Image Quality Assessment
}

\author{
Fatima Eashour and Stephen Pistorius \\ Department of Physics and Astronomy, University of Manitoba \\ Winnipeg, MB, R3T2N2, Canada \\ eashourf@myumanitoba.ca; stephen.pistorius@umanitoba.ca
}

\section{Extended Abstract}

Introduction: To date, there is no gold standard for medical image quality assessment. Commonly used assessment methods are influenced by either manual selection or the algorithmic driven automatic selection of a Region of Interest (ROI) [1-4]. An ROI is used in measurements of the signal mean, standard deviation, and the system response such as an Edge Spread Function. Noise, artifacts and the user's selection of a ROI can affect the interpretation of the system's response [1,2]. Challenges in image quality assessment are more prominent when dealing with imaging systems with poor SNR [5].

Objective and Scope: This research aims to develop and evaluate the feasibility of an analytical medical image quality assessment that requires no user input. This method is intended for the quality assessment of imaging systems during design, commissioning, and routine quality assurance. The approach estimates image degradation factors, applies them to the known object in the image and compares the output with the test image. The comparison is executed in the histogram space, removing the spatial dependence present in ROI-based methods. Since the SNR is a limiting factor of the ability to measure a system's performance, the method was evaluated at varying SNR levels.

Methods: As a feasibility study, planar x-ray imaging was chosen as a medical imaging technique that does not require image reconstruction and can be assumed to be quantum noise limited. The method was tested using Monte Carlo simulated planar images of a simple disk phantom. The method's accuracy and precision were compared to conventional methods of image quality measurement.

Results and Conclusion: As expected, the performance in estimating quality parameters varied as a function of SNR. As the SNR decreased, the noise degradation became more significant than the degradation due to blurring in the cumulative histogram curve, and the number of data points in the fitting process decreased. Aside from the SNR, factors that affected the fit of the proposed cumulative histogram model to the Monte Carlo simulated data included the increased attenuation in proportion to the inverse square of the distance from the diverging beam source. Another factor was the dose fall-off at the edge of the field of view (FOV). The effect of these factors on the accuracy and precision of the results varied and depended on the variation of the phantom background attenuation and the size of the global ROI within the FOV.

The best results were achieved with SNR values of at least $11.0(+0.3,-0.2)$. For these levels an average error in the signal and noise measurements of no more than $0.1(+0.1,-0.1) \%$ was obtained, while the average error in the measurement of the frequency at $10 \%$ Modulation Transfer Function (MTF) was $0.1(+0.2,-0.1)$ cycle/mm. For images with an SNR of less than 10 (or less than $20 \mathrm{~dB}$ ), the results were less promising and the error and uncertainty in noise and spatial resolution measurements were larger than conventional methods. Therefore, despite the benefits of automation offered using this approach, further work is needed to enable this approach to be applied to low dose imaging systems.

\section{References}

[1] A. Carton, D. Vandenbroucke, L. Struye, A. D. A. Maidment, Y. Kao, M. Albert, H. Bosmans, and G. Marchal, "Validation of MTF measurement for digital mammography quality control," Medical Physics, vol. 32, no. 6, pp. 1684-1695, 2005.

[2] S. N. Friedman and I. A. Cunningham, "Normalization of the modulation transfer function: The open-field approach," Medical Physics, vol. 35, no. 10, pp. 4443-4449, 2008. 
[3] K. Gulliksrud, C. Stokke, and A. C. Trægde Martinsen, "How to measure CT image quality : Variations in CTnumbers, uniformity and low contrast resolution for a CT quality assurance phantom," Physica Medica, vol. 30, no. 4, pp. 521- 526, 2014. [Online]. Available: http://dx.doi.org/10.1016/j.ejmp.2014.01.006

[4] L. A. Pierce II, D. W. Byrd, B. F. Elston, J. S. Karp, J. J. Sunderland, and P. E. Kinahan, "An algorithm for automated ROI definition in water or epoxy-filled NEMA NU-2 image quality phantoms," Journal of Applied Clinical Medical Physics, vol. 17, no. 1, pp. 440-456, 2016.

[5] D. R. Gerwe, C. E. Luna, and B. Calef, "Effect of Low SNR on Visual Image Quality," in Imaging and Applied Optics 2014, OSA Technical Digest [online] (Optical Society of America, 2014), paper SM3F.2. 